\title{
Brain activation in the chronic phase of traumatic spinal cord injury
}

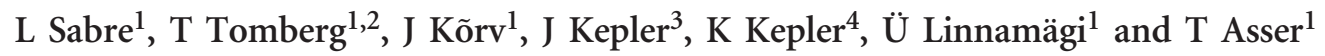

Study design: Cross-sectional clinical study.

Objectives: Plastic changes within cortical areas occur after traumatic spinal cord injury (TSCI). The aim of the study was to assess cortical activation in the chronic phase of TSCI using functional magnetic resonance imaging (fMRI).

Setting: Tartu University Hospital, Tartu, Estonia.

Methods: Ten right-handed patients with paraplegia and 18 healthy controls were studied by fMRI. Individuals performed simple flexion/extension of the right hand fingers and the right ankle during fMRI. The activation volumes, maximum $t$ values ( $T$ max) and centres of gravity (COG) were calculated.

Results: The mean time since trauma was $1848 \pm 1046$ days (range 388-4459). During hand movements, the volume of activation (VOA) in the contralateral primary motor cortex was significantly larger among the TSCI patients who did not recover compared with the controls (4112 vs 2777, $P=0.02$ ). The VOA did not enlarge during the ankle movements (2420 vs $1114, P=0.08$ ). There was a significant relationship between the VOA in Brodmann area 4 (BA4) and American Spinal Injury Association motor score during hand movements $(r=-0.67, P=0.03)$. A positive correlation was found during hand movements in the VOA of BA4 and time since injury $(r=0.62, P=0.05)$.

Conclusions: We found increased cortical activation in the chronic phase of thoraco-lumbar TSCI that may be caused by increased use of upper limbs.

Spinal Cord (2016) 54, 65-68; doi:10.1038/sc.2015.158; published online 22 September 2015

\section{INTRODUCTION}

Traumatic spinal cord injury (TSCI) leads to sensorimotor loss and disruption of autonomic nervous system. ${ }^{1}$ Although TSCI is considered an untreatable condition, several studies in animal models have demonstrated that functional recovery is feasible if any neuronal connection has been survived or is re-established.

The functional reorganisation of the nervous system after injury can be divided into early and long-term changes. ${ }^{2}$ Such changes take place in the area of injury as well as in the brain cortex. The early changes are quite transient and reflect the reinforcement of pre-existing connections. ${ }^{3}$ The studies in patients with TSCI in the acute phase have demonstrated different shifts in the activation and changes in the area of cortical activation. ${ }^{4,5}$

On the other hand, in the chronic phase, new connections are forming and other neural structures take a more leading role in task performance. ${ }^{3}$ Many neuroimaging and mapping studies have also been conducted in the chronic phase of SCI. Unfortunately, the methodology of the studies and the participants have not been homogenous. ${ }^{6}$ In this context, we tried to study cortical activation of the similar patients with chronic TSCI whose neurological deficit bore resemblance. The aim of the study was to evaluate cortical reorganisation depending on the neurological function and time since injury.

\section{PATIENTS AND METHODS}

\section{Participants and tasks}

Ten right-handed patients with TSCI were studied by functional magnetic resonance imaging at a single point of time. The extent of injury was estimated according to the American Spinal Injury Association (ASIA) Impairment Scale (AIS). Five patients were defined as AIS A, one patient had residual sensory function but no motor function in the legs (AIS B, motor complete) and four patients had incomplete lower paraplegia (Table 1). Eighteen controls comparable in age and gender with the patients were also studied.

The patients were chosen from the Estonian TSCI database from 1997 to 2007.7 Therefore, the neurological status from the time of injury to the study was documented. The study was approved by the Research Ethics Committee of the University of Tartu, Estonia. All the participants signed informed consent permission before the study.

All tasks were used in a block design. Motor task of the hand was flexion/ extension of the right-hand fingers and for the foot was flexion/extension of the right ankle. All the patients were able to perform movement by hand. As the majority of patients had severe paraparesis, they were required to try to move their right ankle. The attempt was visually monitored. Hand and foot motor tasks have been run separately.

Length of a block was 10 repetition times (TRs) with the TR of $4 \mathrm{~s}$. Three blocks of resting and three blocks of motor task were carried out alternately, starting with the resting block.

\section{Image acquisition}

Before examination, the participants were informed about the procedure, tasks and length of the examination. Images were obtained on a 1.5 T MR scanner

\footnotetext{
${ }^{1}$ Department of Neurology and Neurosurgery, University of Tartu, Tartu, Estonia; ${ }^{2}$ Radiology Clinic, Tartu University Hospital, Tartu, Estonia; ${ }^{3}$ Medical Technology Service, Pärnu Hospital, Pärnu, Estonia and ${ }^{4}$ Institute of Physics, University of Tartu, Tartu, Estonia

Correspondence: Dr L Sabre, Department of Neurology and Neurosurgery, University of Tartu, 8L. Puusepa Street, Tartu 51014, Estonia.

E-mail: liis.sabre@kliinikum.ee

Received 12 February 2015; revised 8 August 2015; accepted 10 August 2015; published online 22 September 2015
} 
Magnetom Symphony (Siemens Medical Systems, Erlangen, Germany). Prior to the functional scans, high resolution T1-weighted anatomical images were obtained with gradient echo FLASH sequence (TR $=12 \mathrm{~ms}$, echo time $(\mathrm{TE})=5.68 \mathrm{~ms}$, flip angle $15^{\circ}$, resolution $224 \times 256$, voxel size $1 \times 1 \times 1 \mathrm{~mm}^{3}$, 176 sagittal planes). Functional $\mathrm{T}^{*}$-weighted images were obtained using gradient echo EPI sequence $\left(\mathrm{TR}=4 \mathrm{~s}, \mathrm{TE}=50 \mathrm{~ms}\right.$, spin flip angle $90^{\circ}$, resolution $64 \times 64$, voxel size $3 \times 3 \times 3 \mathrm{~mm}^{3}$, slice gap $0.75 \mathrm{~mm}, 36$ axial planes, interleaved scan). Sixty whole brain functional images were acquired for each patient and control while they were performing the motor tasks.

Image processing

Image processing was performed using Statistical Parametric Mapping (SPM8, Wellcome Trust Centre for Neuroimaging, London, UK) software.

Table 1 Clinical data of the patients with TSCI

\begin{tabular}{lcccccc}
\hline $\begin{array}{l}\text { Subject } \\
\text { with TSCl }\end{array}$ & $\begin{array}{c}\text { Age } \\
\text { (years) }\end{array}$ & $\begin{array}{c}\text { Time since } \\
\text { TSCl (days) }\end{array}$ & $\begin{array}{c}\text { Level of } \\
\text { motor } \\
\text { impairment }\end{array}$ & $\begin{array}{c}\text { AIS } \\
\text { grade }\end{array}$ & $\begin{array}{c}\text { ASIA lower } \\
\text { extremity } \\
\text { motorscore }\end{array}$ & $\begin{array}{c}\text { ASIA } \\
\text { sensory } \\
\text { score }\end{array}$ \\
\hline 1 & 29 & 1508 & Th5 & A & 0 & 48 \\
2 & 34 & 1838 & Th9 & A & 0 & 66 \\
3 & 25 & 1761 & Th5 & B & 2 & 88 \\
4 & 27 & 1303 & Th4 & A & 0 & 40 \\
5 & 24 & 1998 & Th5 & A & 0 & 48 \\
6 & 37 & 4459 & Th10 & A & 0 & 70 \\
7 & 25 & 1438 & L3 & D & 42 & 112 \\
8 & 33 & 2272 & Th12 & D & 48 & 105 \\
9 & 33 & 1510 & L3 & C & 16 & 90 \\
10 & 24 & 388 & L3 & D & 40 & 112 \\
\hline
\end{tabular}

Abbreviations: AIS, American Spinal Injury Association Impairment Scale; ASIA, American Spinal Injury Association; TSCI, traumatic spinal cord injury.
The first step of spatial pre-processing was the realignment of functional images, where movement effects were discounted. To maximise the mutual information, high resolution anatomical images were co-registered with functional images. Pre-processing continued with segmentation of high resolution anatomical images, where the Montreal Neurological Institute (MNI) 452 white matter, grey matter and cerebrospinal fluid probability maps were used to yield a parametric description for normalisation. ${ }^{8}$ During normalisation, images were also bias-corrected. Image pre-processing ended with smoothing by $8 \times 8 \times 8 \mathrm{~mm}^{3}$ FWHM (full-width at half maximum) isotropic Gaussian kernel.

Statistical analysis

Modelling was performed with box car functions convolved with canonical hemodynamic response function. Low frequency noise was eliminated by using a 160-s high-pass filter. We used SPM8 to estimate model parameters. Thereafter task vs rest activation was assessed by applying a $t$-test to the parameter estimates, resulting statistical parametric t-maps for each participant. The problems with multiple comparisons were corrected by masking images with the BA masks BA 1-2-3-5 (BA 1, 2, 3 and 5 were analysed as one area), BA 4 and BA 6 from MRIcro ${ }^{9}$ using SPM8 and by applying family-wise error correction with $P<0.05$ and by discounting all clusters smaller than 3 voxels.

From resulting masked t-maps, maximum $t$-test results and Talairach coordinates of the maximum activation were recorded. The total volume of all activated cluster(s) (volume of activation, VOA) in each region of interest (defined by the masked areas) was calculated. As each BA may include many active clusters (in one or in both hemispheres), only the cluster with the highest $t$-test result in each region of interest was taken into account when calculating the geometric centre of gravity. Talairach coordinates of the centre of gravity of the cluster were found using MarsBaR release 0.43. ${ }^{10}$

\section{RESULTS}

The mean age was $29.1 \pm 4.8$ years among the patients and $28.5 \pm 4.2$ among the controls.

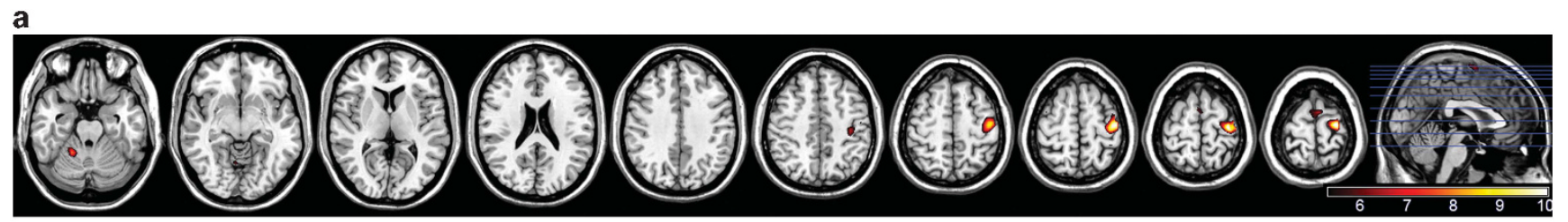

b

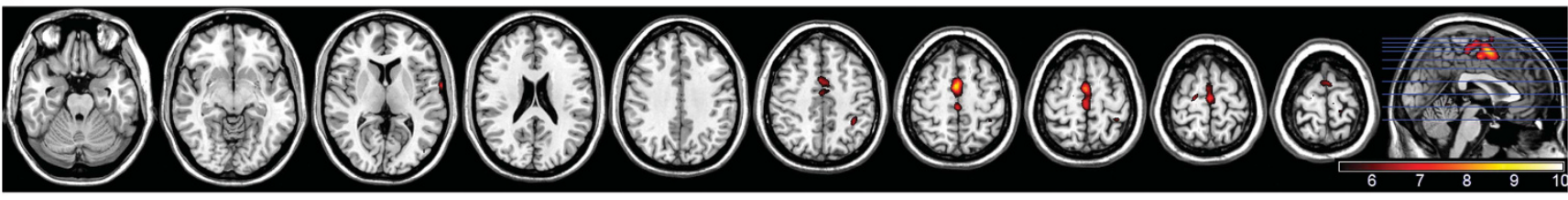

c

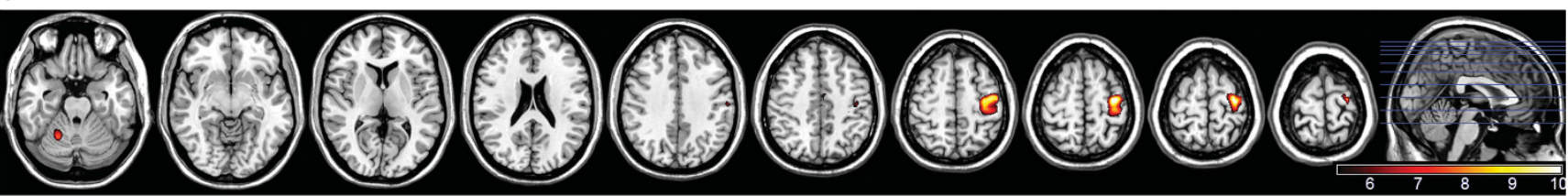

d

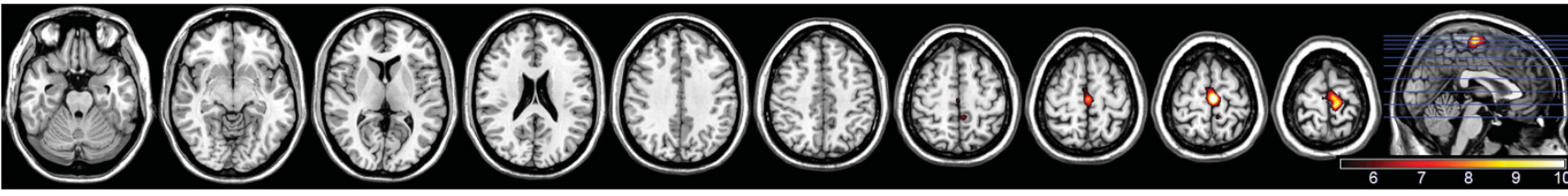

Figure 1 Activation maps for a representative control (control 6): (a) activation map of the right-hand movements; (b) activation map of the right-ankle movements. Activation maps for a representative patient with TSCI (participant 1): (c) activation map of the right-hand movements; (d) activation map of the right-ankle movements. 


\section{Controls}

The mean movement rate of the control individuals was $0.49 \pm 0.11 \mathrm{~Hz}$ during hand movement and $0.50 \pm 0.14 \mathrm{~Hz}$ during ankle movement.

There was no correlation between hand nor ankle movement rate and VOA in BA4. The VOA in the primary motor cortex was not correlated with the age of the controls (hand $P=0.89$, ankle $P=0.60$ ). Figures $1 \mathrm{a}$ and $\mathrm{b}$ show the activation maps during right hand and right ankle movements for a representative control individual.

\section{TSCI patients}

The individual characteristics of the patients are detailed in Table 1.

The mean time since trauma was $1848 \pm 1046$ days (range 388-4459). The mean hand movement rate was $0.50 \pm 0.23 \mathrm{~Hz}$ and there was no significant difference between the SCI patients and the controls $(P=0.58)$. As there was no ankle movement among most of the patients, the correlation could not be calculated.

VOA data during right-hand and right-ankle movements for the representative participant with TSCI are shown in Figures $1 \mathrm{c}$ and $\mathrm{d}$. A statistical comparison between the VOA in BA4, BA1-2-3-5 and BA6 did not reveal any significant difference between the SCI patients and the controls (see Table 2 for BA4). The VOA in BA4 was significantly larger among the patients with motor complete thoracic injury (AIS A or B) compared with the controls $(4112 \pm 1513$ vs $2732 \pm 1035, P=0.02)$.

With respect to maximum $t$-values, the comparison did not show any significant differences between the patients and the controls during the hand movement but $t$-value in the contralateral primary motor cortex was significantly larger among the SCI patients when they moved the ankle (Table 2). The statistical comparison between centre of gravity coordinates obtained for all movements did not reveal any significant differences.

There was a negative correlation between the VOA in BA4 and ASIA motor score during hand movement $(r=-0.67, P=0.03)$. Interestingly, age correlated positively with the VOA in every region of interest during hand movement (BA4 $r=0.76, P=0.01$; BA1-2-3-5 $r=0.80, P=0.006$; BA6 $r=0.80, P=0.01)$. In contrast, no correlation was found during ankle movement (BA4 $r=0.23, P=0.56$; BA1-2-3-5 $r=-0.43, P=0.33$; BA6 $r=0.32, P=0.40$ ).

A positive correlation was also found during hand movement in the VOA of BA4 and time since injury $(r=0.62, P=0.05)$.

\section{DISCUSSION}

Our study concentrated on imaging of cortical reorganisation in the chronic phase of TSCI. The activation maps of the participants with chronic TSCI were generally similar to the control group. Still, when we separated the wheelchair users with motor complete paraplegia from patients who were walking themselves, some differences were revealed. The activation during hand movement was larger among the patients who did not recover. We also found that the VOA in the primary motor cortex was positively correlated with the time since injury when the participants moved the non-affected upper limb, and long-term paraplegics showed a larger extent of activation in every region of interest during hand movement.

Reorganisation depends on the location of the spinal cord lesion and completeness of injury. Different findings have been reported concerning brain activation, but they are lacking uniformity. ${ }^{6}$ Increase in the activation magnitude was reported by several researchers. ${ }^{6,11,12}$ Some studies have not found differences in brain activation, ${ }^{6,13}$ and two studies have reported reduced activation in individuals with TSCI. ${ }^{14,15}$

Most of the studies have been performed in the chronic phase of injury. Jurkiewicz et al. ${ }^{5}$ studied patients with cervical TSCI during the first year after the injury. They found progressive enlargement of activation in the primary motor cortex and decreased activation in the associated cortical areas when the patients recovered. ${ }^{5}$ When these authors studied four tetraplegic individuals whose paralysis persisted, they saw extensive activation in the associated motor areas in the beginning of the study that progressed toward no activation by the end of the first year. ${ }^{16}$ This confirms our findings that majority of substantial changes occur in the acute phase of injury.

Curt et al. ${ }^{12}$ reported similarly to us that their patients in the chronic phase of thoracic or lumbar level TSCI had increased brain activation when they moved their intact limb. This indicates that the longer the time the patient has trained his/her hands, the larger the activation of the cortex will become. The explanation for the correlation with age could be compensatory over-usage of hands among the older people.

There were some limitations in our study. The main limitation is the small number of patients. Although the total number of participants was 10 , the group was not homogeneous. Six of the patients had complete motor lesion and four had some improvement after TSCI. The unfavourable side of cortical reorganisation may be autonomic dysreflexia and neuropathic pain. ${ }^{3}$ Chronic pain is a

Table 2 Analysis of the volumes of activation, maximum $t$-values and centres of gravity in the contralateral primary motor cortex (Brodmann area 4) in patients with $\mathrm{TSCl}$ and controls

\begin{tabular}{|c|c|c|c|c|c|c|c|}
\hline Paradigm & & & $V O A(m m 3)$ & Max t-value & $x$ & $y$ & $z$ \\
\hline \multirow{3}{*}{ Hand } & & s.d. & 1755 & 3.36 & 2.9 & 2 & 2.7 \\
\hline & Controls & Mean & 2732 & 10.9 & -38.9 & -21.9 & 58.2 \\
\hline & & s.d. & 1035 & 2.5 & 1.6 & 1.9 & 2.3 \\
\hline \multirow{4}{*}{ Ankle } & & s.d. & 2567 & 2.25 & 5.3 & 3.4 & 6.5 \\
\hline & Controls & Mean & 1114 & 7 & -6.5 & -25.7 & 68.6 \\
\hline & & s.d. & 1277 & 1.31 & 4.4 & 5.03 & 3.8 \\
\hline & $t$ test & $P$ & 0.08 & 0.04 & 0.57 & 0.72 & 0.14 \\
\hline
\end{tabular}

Abbreviations: SCl, spinal cord injury; s.d., standard deviation; TSCI, traumatic spinal cord injury; VOA, volume of activation. 
common consequence of TSCI and significant changes in a number of cortical regions, including thalamus, prefrontal cortex, premotor cortex, insular cortex and amygdala, take place after TSCI. ${ }^{17}$ Although the patients were neurologically assessed during the study, we did not ask about the pain at the moment of the imaging. Finally, we did not image the spinal cord, so it could not be possible to correlate the size of the damaged area with cortical reorganisation. ${ }^{18}$

Our study demonstrates the effect of hand training in chronic paraplegics. The severity of disability is quite stable in the chronic phase of TSCI. Spontaneous functional recovery may take place in $40 \%$ of TSCI patients in the acute phase. ${ }^{19}$ Although continuous cortical reorganisation has been pointed out, ${ }^{2}$ the time frame is important. ${ }^{4}$ The greatest gains in motor skills occur in the first 3 months. ${ }^{4,20}$ However, neuroplasticity will not stop in the chronic phase of TSCI. It may manifest by negative consequence like autonomic dysreflexia or neuropathic pain. Therefore, physiotherapy providing exercises and modifying existing function is important to avoid maladaptive and promote adaptive plastic changes.

\section{CONCLUSIONS}

The changes in cortical activation of the non-affected muscles long after TSCI demonstrate the continuous process of reorganisation. Our study confirms that most cerebral adaptions to TSCI take place in the acute phase. Differences in the activation patterns between individuals with chronic TSCI and controls indicate the effect of training. To optimise the management and rehabilitation, imaging biomarkers are most useful in the acute phase.

\section{DATA ARCHIVING}

There were no data to deposit.

\section{CONFLICT OF INTEREST}

The authors declare no conflict of interest.

\section{ACKNOWLEDGEMENTS}

The study was supported by the Estonian Science Foundation research grant No. 7868 and by the Estonian Institutional Research grant IUT2-4.
1 Dietz V, Curt A. Neurological aspects of spinal-cord repair: promises and challenges. Lancet Neurol 2006; 5: 688-694.

2 Chen R, Cohen LG, Hallett M. Nervous system reorganization following injury. Neuroscience 2002; 111: 761-773.

3 Pascual-Leone A, Amedi A, Fregni F, Merabet LB. The plastic human brain cortex. Annu Rev Neurosci 2005; 28: 377-401.

4 Sabre L, Tomberg T, Kõrv J, Kepler J, Kepler K, Linnamägi Ü et al. Brain activation in the acute phase of traumatic spinal cord injury. Spinal Cord 2013; 51: 623-629.

5 Jurkiewicz MT, Mikulis DJ, Mcllroy WE, Fehlings MG, Verrier MC. Sensorimotor cortical plasticity during recovery following spinal cord injury: a longitudinal fMRI study. Neurorehabil Neural Repair 2007; 21: 527-538.

6 Kokotilo KJ, Eng JJ, Curt A. Reorganization and preservation of motor control of the brain in spinal cord injury: a systematic review. J Neurotrauma 2009; 26: 2113-2126.

7 Sabre L, Pedai G, Rekand T, Asser T, Linnamägi U, Kõrv J. High incidence of traumatic spinal cord injury in Estonia. Spinal Cord 2012; 50: 755-759.

8 Mazziotta J, Toga A. A probabilistic atlas and reference system for the human brain: International Consortium for Brain Mapping (ICBM). Philos Trans $R$ Soc Lond B Biol Sci 2001; 356: 1293-1322.

9 Rorden C, Brett M. Stereotaxic display of brain lesions. Behav Neurol 2000; 12: 191-200.

10 Brett M, Anton JL, Valabregue R, Poline JB. Region of interest analysis using an SPM toolbox [abstract]. Neurolmage 2002; 16: S497.

11 Bruehlmeier M, Dietz V, Leenders KL, Roelcke U, Missimer J, Curt A. How does the human brain deal with a spinal cord injury? Eur J Neurosci 1998; 10: 3918-3922.

12 Curt A, Alkadhi H, Crelier GR, Boendermaker SH, Hepp-Reymond MC, Kollias SS. Changes of non-affected upper limb cortical representation in paraplegic patients as assessed by fMRI. Brain 2002; 125: 2567-2578.

13 Halder P, Curt A, Brem S, Lang-Dullenkopf A, Bucher K, Kollias S et al. Preserved aspects of cortical foot control in paraplegia. Neuroimage 2006; 31: 692-698.

14 Cramer SC, Lastra L, Lacourse MG, Cohen MJ. Brain motor system function after chronic, complete spinal cord injury. Brain 2005; 128: 2941-2950.

15 Sabbah P, de SS, Leveque C, Gay S, Pfefer F, Nioche C et al. Sensorimotor cortical activity in patients with complete spinal cord injury: a functional magnetic resonance Imaging study. J Neurotrauma 2002; 19: 53-60.

16 Jurkiewicz MT, Mikulis DJ, Fehlings MG, Verrier MC. Sensorimotor cortical activation in patients with cervical spinal cord injury with persisting paralysis. Neurorehabil Neural Repair 2010; 24: 136-140.

17 Gustin SM, Wrigley PJ, Siddall PJ, Henderson LA. Brain anatomy changes associated with persistent neuropathic pain following spinal cord injury. Cereb Cortex 2010; 20: 1409-1419.

18 Freund P, Weiskopf N, Ward NS, Hutton C, Gall A, Ciccarelli O et al. Disability, atrophy and cortical reorganization following spinal cord injury. Brain 2011; 134: 1610-1622.

19 Ding Y, Kastin AJ, Pan W. Neural plasticity after spinal cord injury. Curr Pharm Des 2005; 11: 1441-1450.

20 Cadotte DW, Fehlings MG. Visualizing plasticity and repair in the injured CNS. Nat Rev Neurol 2013; 9: 546-547. 